DOI: $10.20472 / E F C .2018 .010 .003$

\author{
ANDRÁS BEBES \\ Government Debt Management Agency Pte. Ltd., Hungary \\ DÁVID TRAN \\ Government Debt Management Agency Pte. Ltd., Hungary \\ LÁSZLÓ BEBESI \\ UniCredit Hungary, Hungary
}

\title{
OPTIMIZING THE HUNGARIAN GOVERNMENT DEBT PORTFOLIO
}

\begin{abstract}
:
We construct an optimal debt portfolio model with the purpose of optimizing the Hungarian government debt portfolio. To analyze the characteristics of the costs and corresponding risk factors of the Hungarian debt portfolio we simulate issuances of chosen instruments on a specified time horizon. We apply a multiobjective optimization scheme to construct compositions of financing that minimize the costs and risks of the debt portfolio. Our purpose is to find the set of Pareto-optimal solutions that minimize expected costs, volatility of costs and refinancing risks while maximizing average time to re-fixing. The results of the multiobjective optimization can be used to help in constructing a medium term debt management strategy.
\end{abstract}

\section{Keywords:}

Multiobjectiove Optimization, Portfolio Optimization, Government Debt Management

JEL Classification: C61, G17, H63 


\section{Introduction}

In this paper we construct an optimal debt portfolio model with the purpose of optimizing the Hungarian government debt portfolio. The main objective of government debt management is to create a structure of financing that minimizes costs while keeping risks at an acceptable level. These two goals are contradictory as lower costs can usually be achieved only by taking on higher risks. Therefore, a single optimal debt portfolio does not exist. However, we can aim to attain a set of Pareto-optimal solutions that can be considered by the debt manager as feasible alternatives.

Consequently, the purpose of the model is to help the Hungarian Government Debt Management Agency in creating a medium term (5 years) debt management strategy.Several countries have developed stochastic optimal portfolio models for debt management purposes. Notable examples include Canada (Bolder and Deeley, 2011), Italy (Adamo et al., 2004; Consiglio and Staino, 2012), Sweden (Bergström et al., 2002), Turkey (Balibek and Memis, 2012) and the United Kingdom (Pick and Anthony, 2006).

In order to contribute to the decision making process, our model needs to predict the costs and risks associated with any given financing structure on a 5-year horizon (20182022). This requires us to price all outstanding government debt instruments as well as those to be issued in the future. We use the results obtained using our Markov regime switching state-space model (Bebes et al., 2018)for this purpose.As we intend to minimize costs and risks, we use an evolutionary multiobjective optimization algorithm to find Pareto-optimal solutions. Like traditional optimization methods, a number of iterations (repeated calculations of the objective function) is required to reach an optimum. However, contrary to conventional methods, we use a large set of inputs at every iteration instead of a single point or vector in order to obtain a set of Pareto-optimal solutions.

The structure of this paper is as follows: Section 2 introduces the factual and forecasted data that provide the background for optimization. This includes the results of the Markov regime switching state-space Model (Bebes et al., 2018), the outstanding government debt portfolio and the specifications of debt instruments to be issued in the future. Section 3 and 4 deals with the various cost and risk metrics employed in the objective function of the optimization. Section 5 gives an overview of the optimization procedure including the objective function, the optimization algorithm and the simulated debt issuance. Section 6 details the results of the optimization, presenting optimal financing structure and cost-risk frontier results. Section 7 concludes.

\section{Data}

In this section we provide insight to the specifics of our data that we use for our optimal portfolio model.It is driven by the Markov regime switching state-space model developed by Bebes, Tran and Bebesi (2018). 


\subsection{The Markov regime switching state-space model and forecast results}

As detailed in (Bebes et al., 2018) our historical dataset includes monthly Hungarian and Euro area yield curve points, Hungarian CDS spread curve points.It also includes Hungarian and Eurozone CPI as well as EUR/HUF exchange rate data from October 2008 to December 2017 for a total of $n=111$ observations, giving us roughly 10 years of historical data.

For the optimal portfolio model we need a forecast of our underlying factors between the 2018-2022 5-year timeframe. The state-space representation of our model can be written as

$$
\begin{gathered}
Y_{t}=H_{t} X_{t}^{\prime}+\theta_{t}, \\
X_{t+1}=\mu_{S_{t}}+X_{t} F_{S_{t}}+\varepsilon_{t}, \\
{\left[\begin{array}{c}
\theta_{t} \\
\varepsilon_{t}^{\prime}
\end{array}\right] \sim N\left(0, \quad\left(\begin{array}{cc}
R_{S_{t}} & 0 \\
0 & Q_{S_{t}}
\end{array}\right)\right),}
\end{gathered}
$$

where $Y_{t}$ denotes the vector of observations, while $X_{t}$ contains the unobserved state variables, our factors at time $t . S_{t}$ denotes an unobserved, discrete-valued 2-state Markov-switching variable. Our prior assumption is that the economy switches between two regimes. One represents economic stability or growth, while the other represents a crisis period.

Figure 1: Fan charts of selected yields, CDS spreads, CPI rates and exchange rates with 5$95 \%$ confidence intervals
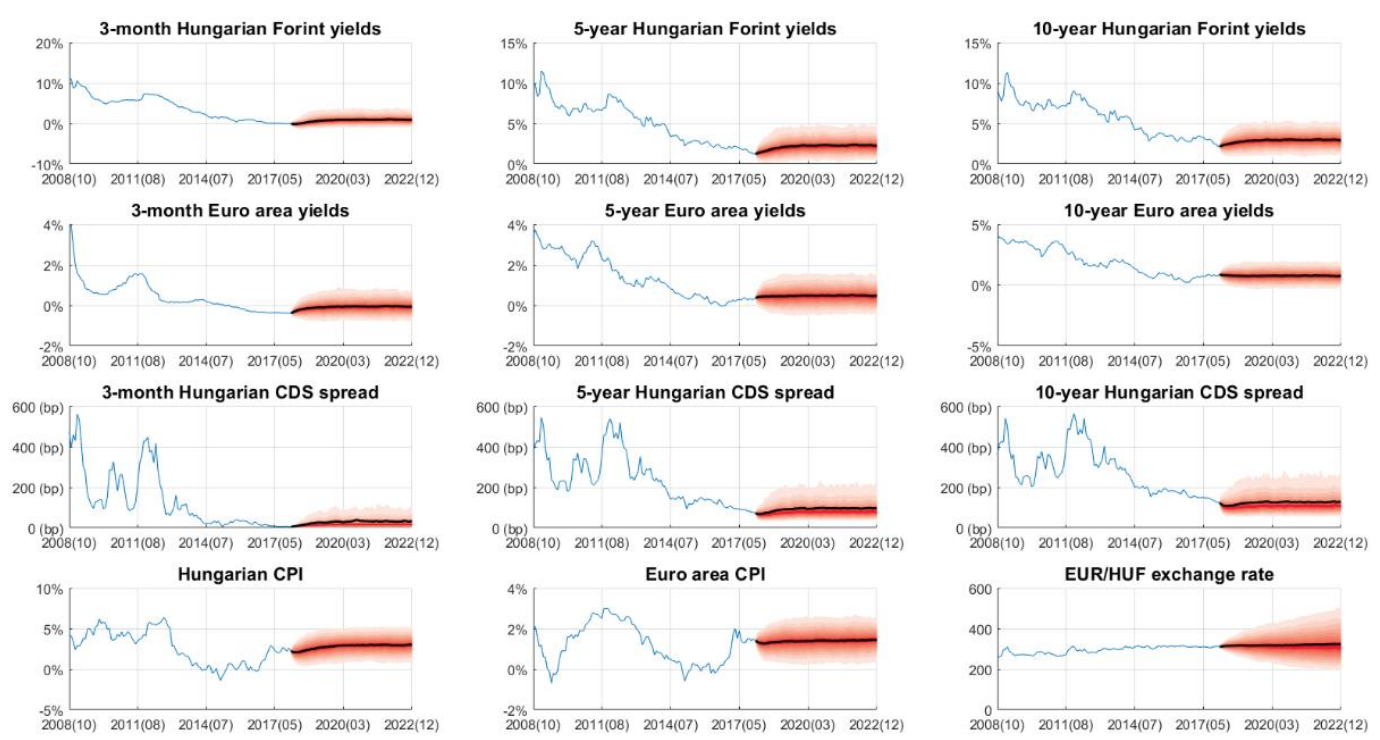

Source: 5-year forecasts based on fitting results (Bebes et al., 2018) 
For a more detailed description of the underlying Markov regime switching state-space model, and the fitting of the model please refer to (Bebes et al., 2018).

Figure 1 shows our forecast of the main yield curve points and macro factors. The Hungarian short rate is expected to rise slowly to the $1 \%$ level, while long rates are also predicted to rise. According to our forecast, Euro area rates are mostly expected to stagnate, with the exception of the short rate converging to zero. The overall volatility is expected to be low. The fan charts also show that risks leading to the depreciation of the HUF as well as risks of higher interest rates are significantly higher than vice versa. CDS spreads in particular are especially vulnerable and can top 500bps in a crisis situation.

\subsection{Issuance characteristics}

To analyze the characteristics of the costs and corresponding risk factors of the Hungarian debt portfolio we simulate issuances of chosen instruments on the specified time horizon. We categorize all portfolio instruments into three separate groups, namely the wholesale, the FX, and the retail debt. There are several model instruments to choose from in each market segment:

1) Wholesale segment:

- 3-, 5- and 10-year fixed interest rate Government Bonds

- 5-year floating interest rate Government Bond

- 12-month Discount Treasury Bill

2) FX segment:

- 5- and 10-year fixed and floating interest rate, Euro denominated Bonds

3) Retail segment:

- 1-year Hungarian Government Security

- Premium and Bonus Hungarian Government Securities

All model instruments share the same characteristics and properties as those issued by the Hungarian DMO. The range of model instruments can be easily changed by adding or removing instrument types.

The share of each retail instrumentin the retail segment is fixed at the starting share for the time horizon. The premia of the retail debt instruments are connected to the level of the short rate at every trajectory. It is assumed, that retail investors expect a higher premium when the short rate is at a low level. A higher debt-renewal willingness is also assumed for the retail segment based on the DMO's experience.

In the model Government Bonds are issued quarterly, with the opportunity of a monthly reopening, while Treasury Bills are issued each month. FX Bonds are issued yearly with 
a trajectory dependent choice for the issuance month ${ }^{1}$, and are not reopened. Retail instruments (except the 1-year Hungarian Government Security, which is issued every month) are issued at the start of every calendar year, with the opportunity of a monthly reopening.

The initial portfolio to start the simulation is identical to the actual government debt portfolio, with the exception that all actual instruments are listed as one of the above model instruments. Thus, the model is able to generate accurate and realistic results that can be used directly to set benchmarks for the government debt.

\section{Cost factors}

Cost factors can be divided into 4 groups. The most prominent cost factor is the interest expenditure, which is the cumulated sum of the coupon payments of all instruments. The second cost factor is the issuance price discount or premium (loss or gain). Treasury Bills do not pay coupons whereasall other instruments are issued exactly at, or closely toparin the model.The third cost factor is the currency cost, which boils down from the deviation of the EUR/HUF exchange rate. In accordance with the strategic goal of the Hungarian $\mathrm{DMO}$, all foreign liabilities are either issued in, or swapped into Euro. The model calculates all costs in HUF (based on Hungarian accounting rules), therefore currency cost is a key factor in determining the optimal share of FX debt in the portfolio. The fourth cost factor is the so-called liquidity premium, which ariseswhen excessive issuance of government bonds or treasury bills is needed to fulfill the financing requirements. We address this issue in Section 4.3.

We call a series of random numbers substituted into the transition equation of our model a trajectory. A financing composition is a weighting of the model instruments, summing to 1 , while aiming for the theoretical composition of the portfolio, as time $t \rightarrow \infty$.

All costs are aggregated in HUF over the time horizon on every trajectory using accrual accounting. Such time-proportional accounting applies for all coupon payment dates in the timeframe, for all instruments. Smoothing the costs on the time horizon makes the financing compositions (and different time horizons) comparable to each other. It also mitigates the importance of whether a payment date is before or after the model's timeframe.

If all the simulated trajectories are taken into account, we get the stochastic distribution of the costs. We call the expected cost of the stochastic distribution of the cumulated costs the cost of the specific financing composition.

\footnotetext{
${ }^{1}$ In practice, the Government Debt Management Agency has a degree of freedom in deciding when to issue FX debt. This is modeled by a single issuance per calendar year. In the model, FX debt is issued in the first month where the level of Euro area yield curve declines for two months in a row, or in December if the criterion is not met throughout a given calendar year.
} 


\section{Risk factors}

To measure the risks rising from the volatility of the yield curve, we calculate the standard deviation, the Cost-at-Risk and the Conditional Cost-at-Risk at different confidence levels on the stochastic distribution of costs (basically an implementation of the Monte Carlo simulation for calculating $\mathrm{VaR}$ or $\mathrm{CVaR}$ ). The corrected sample standard deviation is well known. Let $c_{1, s} \leq c_{2, s} \leq \cdots \leq c_{r, s}$ denote the ordered costs of $r$ trajectories based on $s$ financing composition, then we calculate the Cost-at-Risk at $0<\alpha<100$ confidence level

$$
\operatorname{CaR}_{\alpha}(s)=c_{\left\lfloor r \frac{\alpha}{100}\right\rfloor}
$$

while the Conditional Cost-at-Risk at $0<\alpha<100$ confidence level

$$
\operatorname{CCaR}_{\alpha}(s)=\frac{1}{\left\lfloor r\left(1-\frac{\alpha}{100}\right)\right\rfloor+1} \sum_{i=\left\lfloor\frac{\alpha}{100}\right\rfloor}^{r} c_{i} .
$$

The Cost-at-Risk at confidence level $\alpha$ tells us that based on the worst $(100-\alpha) \%$ of scenarios how much the minimum cost will be on the chosen timeframe, while the Conditional Cost-at-Risk at confidence level $\alpha$ tells us about the expected costs of the worst $(100-\alpha) \%$ of scenarios on the chosen timeframe.

Although measuring the deviation of costs is crucial, there are several other metrics to assess risks. We address this issue in the following sections.

\subsection{Interest rate sensitivity}

Unexpected changes in the yield curve may cause increase in the interest rate expenditures, and therefore it is crucial to measure interest rate sensitivityfrom a debt management perspective. Short-term or variable interest rate instruments are considered riskier than long-term or fixed rate instrumentsin this aspect. Duration and Average TimetoRe-fixing (ATR) can be used to capture the exposure of the debt portfolio to changes in interest rates.

The ATR of a fixed interest rate instrument is equal to its time to maturity, while for a floating rate instrument it measures the time until its next coupon re-fixing. Thus the ATR of the debt portfolio measures how long on average it takes for the interest rate changes to be incorporated into the costs of the overall debt. Let

$$
a t r_{t}=\frac{\sum_{i=1}^{N} \zeta_{t, i} v_{t, i}}{\sum_{i=1}^{N} v_{t, i}}
$$

denote the ATR of the debt portfolio at time $t$, with $N$ instruments present in the portfolio. $\zeta_{t, i}$ denotes the time to the next coupon fixing at time $t$ for instrument $i$, while $v_{t, i}=$ $F V_{i} P_{t, i}\left(\frac{100-\sum_{k \leq t} C_{k, i}}{100}\right)$, where $F V_{i}$ is the Face Value of instrument $i, P_{t, i}$ denotes the pieces of instruments $i$ issued by the DMO at time $t$, and $C_{k, i}$ is the capital repaid at time $k$ of 
instrument $i$ given in percentage points. A higher value of ATR indicates a higher immunity against interest rate changes, while low values indicate that a high share of the debt portfolio will be subject to interest rate changes in the near future, thus signaling a higher exposure to interest rate risk.

\subsection{Refinancing risk}

The risk rising from the maturity structure of a given portfolio is called refinancing risk. Refinancing risk measures the share of debt portfolio maturing in a given time period. Generally, in periods of turbulence or high volatility, market liquidity typically dries up, making the renewal of maturing debt expensive, or, in an extreme case, impossible.Therefore, it is vital for debt management to smooth the maturity structure, and thus avoid maturity peaks.

Average Time-to-Maturity (ATM) measures how long it takes on average to renew outstanding debt.Let

$$
\operatorname{atm}_{t}=\frac{\sum_{i=1}^{N} \eta_{t, i} v_{t, i}}{\sum_{i=1}^{N} v_{t, i}}
$$

denote the ATM of the outstanding debt portfolio at time $t$, with $N$ instruments present in the portfolio. $\eta_{t, i}$ denotes the time to maturity at time $t$ for instrument $i$, while $v_{t, i}$ is the same as above. Refinancing risk is lower when ATM is higher, as it means a lower renewal rate in the short term.

The refinancing ratio measures the share of the debt portfolio to be renewed on a given time horizon.Let

$$
\operatorname{refin}_{t, \lambda}=\frac{\sum_{i=1}^{N} v_{t, i} \mathbf{1}_{\left\{\eta_{t, i} \leq \lambda\right\}} l_{i}}{\sum_{i=1}^{N} v_{t, i}}
$$

be the refinancing ratio of the outstanding debt portfolio at time $t$, with time horizon $\lambda$. $\mathbf{1}_{\left\{\eta_{t, i} \leq \lambda\right\}}$ denotes the indicator function of whether instrument $i$ at time $t$ matures within the chosen time horizon, and $l_{i}=1 / 10$ if $i$ is retail, while 1 in every other case. We assume and years of experience at the Hungarian Government Debt Management Agency supports - that retail investors' willingness to renew their government securities holdings tend to be higher than wholesale, especially in times of high volatility (as mentioned before, retail rates are higher than wholesale, especially when wholesale rates are at a low level. Thus, for retail investors, holding retail instruments is still worth it even in periods of higher volatility).

Keeping the refinancing ratio at a low level stabilizes the debt service, increases trust in investors and supports higher ratings given by credit rating agencies. 


\subsection{Funding liquidity considerations}

It is clear that meeting the financing needs by issuing in only one or two instruments is impossible due to limits of market demand, and it is also clear that excess issuance comes with a cost. Avoiding financing compositions that encourage excess issuance is desired by the DMO. The introduction of so-called liquidity constraints and liquidity premium arises as a possible solution to handle the cases of excess issuance.

We turn to the Hungarian DMO auction database to calculate issuance limits at a given time with the use of market demand. We calculate two values for every wholesale instrument. One representing the maximum amount to be issued at a given time at normal 'market' price, which we call 'liquidity threshold', while 'liquidity constraints' are introduced as the maximum available amount of an instrument to be issued at a given time. 'Liquidity premium' is introduced as the cost premium for the DMO to be paid for the amount issued above the liquidity threshold.

We assume that T-Bills do not have a liquidity constraint.In practice, investors tend to buy short-term instruments even in periods of high volatility, although with a significant liquidity premium. Therefore, T-Bills can be viewed as safety instruments in distress times when financing requirementscannot be met with the issuance of other instruments.

We introduce an indicator similar to the liquidity risk premium of Drehmann and Nikolaou (2013). Our indicator measures the difference between the rejected bids and the marginal rate. Let $E\left(h_{t}\right)$ be the expected marginal rate of the auction held at time $t$. Let

$$
\iota_{b, i, t}=\left(\rho_{b, i, t}-E\left(h_{t}\right)\right) v_{b, i, t}
$$

be the adjusted rejectedbidon the auction held at time $t$, by the auction participant $i \in$ $\{1,2, \ldots, N\}$. The bid $b \in\{1,2, \ldots, B\}$ stands for the amount $v_{b, i, t}$ and rate $\rho_{b, i, t}$, and $E\left(h_{t}\right)<$ $\rho_{b, i, t}$. Which means that $\iota_{b, i, t}$ is the premium expected by the bidder for the buying of an additional $v_{b, i, t}$ amount above the marginal rate. The liquidity premium at time $t$ is the aggregate of the adjusted rejected bids

$$
l p_{t}=100 \frac{\sum_{i=1}^{N} \sum_{b=1}^{B} \iota_{b, i, t}}{E\left(\bar{v}_{t}\right)}
$$

in basis points, where $\bar{v}_{t}$ denotes the cumulated amount of unsuccessful bids. Liquidity premium thus gives us a quantified value of the investor's willingness of providing additional liquidity. The liquidity premium can be quantified for all instruments and regimes, and then used to measure excess issuances.

\section{Optimization}

The purpose of optimization is to construct compositions of financing that minimize the costs and risks of the debt portfolio. We chose three different risk metrics: standard deviation of the costs, 1-year refinancing ratio and ATR. We intend to maximize the latter 
and minimize the former two as well as the expected costs. These goals are contradictory, therefore finding the set of Pareto-optimal solutions is our objective.

To achieve this, we use a multiobjective optimization algorithm applying evolutionary heuristics to the problem. We implement the algorithm of Sun, Zhu and Cai (2017). The algorithm finds the corner solutions first and fills the optimization space afterwards. The output of the optimization algorithm is a set of Pareto-optimal solutions. A twodimensional Pareto-front can then be established using posterior weighting of the risk metrics, expressing the preferences of the decision maker. Using this method, we can produce a traditional mean-variance efficient frontier or plot the expected costs versus an arbitrarily weighted composite risk measure.

Similar to traditional computational optimization, our implementation relies on multiple iterations (repeated evaluations of the objective function) to approach the optimum. However, to attain multiple Pareto-optimal solutions, we continuously work with a set of inputs, consisting of a large amount of financing compositions. Financing compositions constitute the input of the issuance algorithm.

The objective function $G$ can be mathematically expressed as:

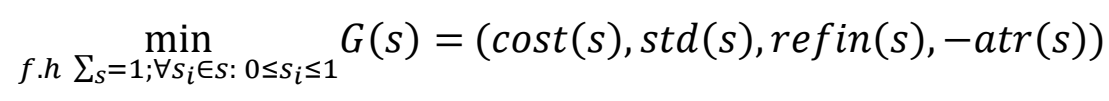

where $s$ is a financing composition, serving as an input for the issuance algorithm. In the first iteration of the optimization process, each financing composition is randomized, taking a uniform distribution on the simplex of the appropriate dimension.

The issuance algorithm is an essential part of the optimization process, connecting input (financing composition) and the output (costs and risks). Issuances are simulated with a monthly frequency. The horizon of the simulation is the first calendar year after the start plus 5 years, adding up to a total of 60-71 months.

Now let $d$ be the number of simulated months, and $\mathrm{v}=\left[v_{0} v_{1} v_{2} \ldots v_{d}\right]^{\prime}$ the total stock of debt instruments we want to attain with $v_{0}$ being the initial stock. Using the vector of yearly projected deficits, we create a monthly $\mathrm{f}=\left[f_{1} f_{2} \ldots f_{d}\right]^{\prime}$ vector. We can then recursively calculate $v_{i}=v_{i-1} f_{i},(i=1,2, \ldots, d)$. We also create a $\mathrm{v}^{\text {max }}=1,05 \mathrm{v}$ maximal stock vector, and a

$$
v_{i}^{\min }=\left\{\begin{aligned}
v_{i}(0,94+0,01 i), & i \leq 5 \\
v_{i}, & i>5
\end{aligned}\right.
$$

minimal stock vector.

The maximal and minimal stock vectors are required due to liquidity constraints and smoothing. Should a large amount of stock mature at the start of the simulation, we can avoid issuing Treasury Bills for liquidity instead of moving towards the desired structure of 
financing. Similarly, a 5\% extra issuance over the required stock allows a buffer in case of a possible eventual crisis and simulates pre-financing strategies employed by DMOs.

If we intend to reach the desired composition of debt in $m$ months, let $\mathrm{w}=\left[w_{1} w_{2} \ldots w_{d}\right]^{\prime}$ be a vector of weights calculated by:

$$
w_{i}=\left\{\begin{array}{rr}
i / m, & i<m \\
1, & i \geq m
\end{array}\right.
$$

Furthermore, let $p$ denote the shares of the initial portfolio and $T$ ad $\times \mathrm{n}$ matrix, where

$$
T_{i j}=v_{i}\left(\left(1-w_{i}\right) p_{j}+w_{i} s_{j}\right) \text {. }
$$

The value of $T_{i j}$ gives the desired stock of instrument $j$ for month $i$.

To calculate the net issuance of instruments for month $i$, let $t$ denote the $i$-th row of $T$ and $u$ denote the current stocks of instruments at month $i$. The net issuance vector $x$ is the result of

$$
\begin{gathered}
\underset{x}{\operatorname{lexmax}}\left(\min \left(\frac{x+u}{t}\right)\right) \\
f . h .: \quad x \geq 0, \\
x \leq \max (0, t-u), \\
L x \leq b, \\
1_{n}^{\prime}(x+u) \leq \max \left(v_{i}^{\max }, 1_{n}^{\prime} u\right),
\end{gathered}
$$

where $b$ is the vector of liquidity constraints and $L$ is the corresponding summary matrix. We use 6 different liquidity constraints: retail, FX, Treasury Bill and 3/5/10-year Hungarian Government Bond.

The so-called lexicographic maximization, inspired by the Constrained Equal Losses rule (Fleiner and Sziklai, 2012), can be interpreted as trying to maximize the smallest share of the actual versus desired stock. If it can be accomplished in multiple ways, we maximize the second smallest share and so on.

Should $1_{\mathrm{n}}^{\prime}(\mathrm{x}+\mathrm{u}) \geq v_{i}^{\text {min }}$ remain unfulfilled, meaning that the minimal total stock is not reached, we finance the remaining value by issuing Treasury Bills, even if its liquidity constraint is exceeded. This simulates emergency borrowing at a significantly higher cost.

\section{Final results}

In this section we present and examine the results of the optimization. We initialized the optimization procedure by randomly generating 5000 financing compositions and refined them through 1000 iterations. Costs and risks were aggregated from December 2017 to December 2022. 
Figure 2 shows the optimal financing compositions given an equal weighting of standard deviation, ATR and 1-year share of refinancing. The composite risks were calculated by individually standardizing the 3 risk measures over the 5000 financing compositions and adding them together.

\section{Figure 2: Optimal compositions of financing, composite risk based on $1 / 3$ standard deviation - 1/3 ATR - 1/3 share of 1-year refinancing}

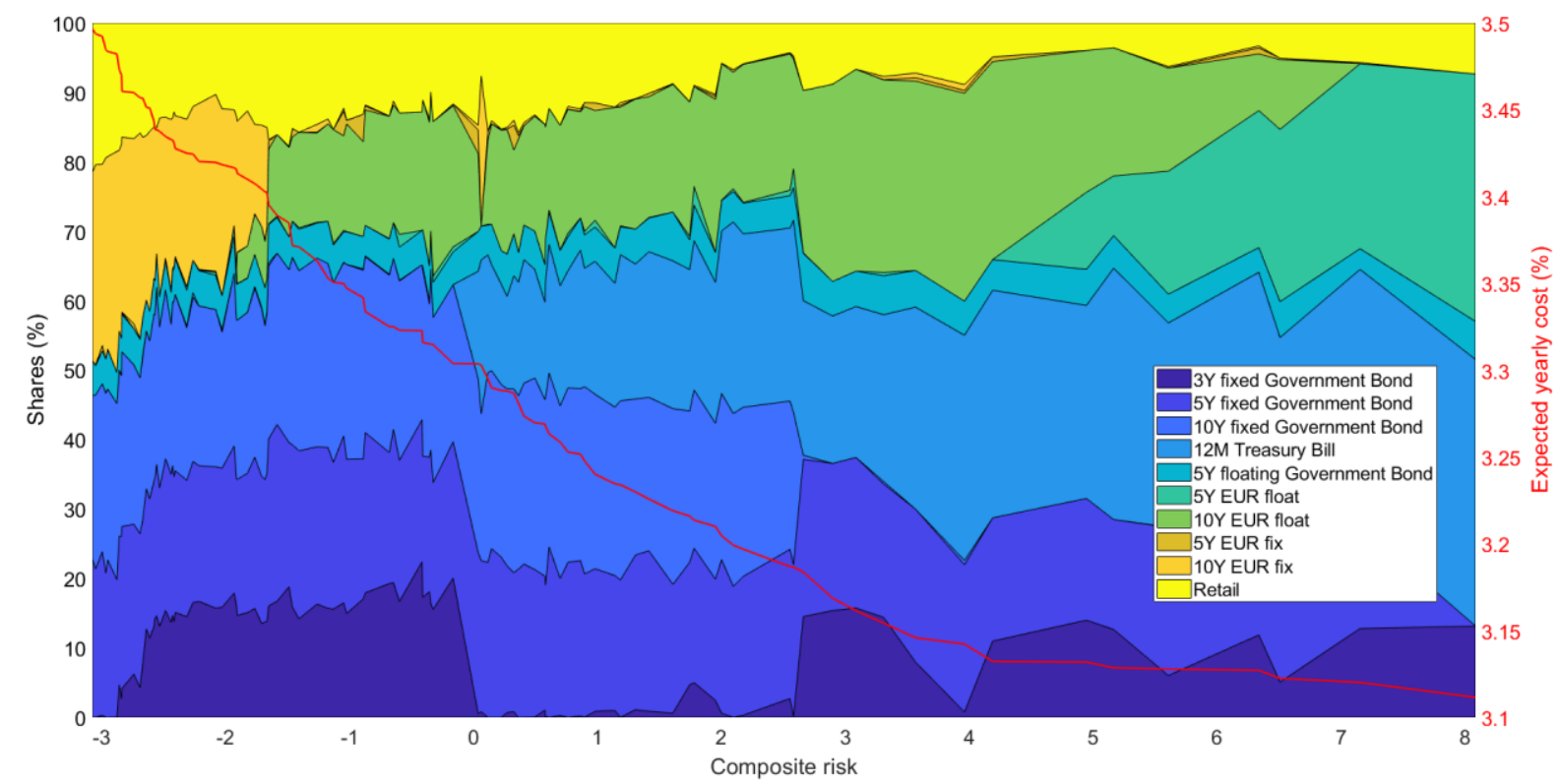

Source: Own calculations

The expected yearly costs of the optimal compositions of financing range from $3.1 \%$ to $3.5 \%$. We can make several observations by examining Figure 2 .

First, bonds with a higher tenor, perceived to carry a lower risk, have a noticeably higher share in lower risk financing structures. On the other hand, a high share of Treasury Bills or floating rate $\mathrm{FX}$ bonds can reduce the expected costs, but at a significantly higher risk. The cost-risk trade-off is easily seen.Second, Figure 2 supports our expectations of the retail program. The costs of financing compositions with a high share of retail instruments is higher, but their addition to the financing mix is a significant mitigating factor on risks. The pricing of retail instruments is in the jurisdiction of the debt manager and they have a higher reinvestment rate as opposed to wholesale instruments, increasing the sustainability of the retail program. Third, a certain level of FX debt is desirable to lower the expected costs. Choosing longer maturity FX debt is feasible and can increase the portfolio ATR while reducing refinancing risk. The high exchange rate risk is certainly a drawback, but it is mitigated by diversifying the rest of the debt.Fourth, the share of 5year floating rate Hungarian Government Bond reaches its theoretical limit set by liquidity constraints in almost every financing composition. Therefore, we can conclude that its lower refinancing risk and longer maturity make it a worthwhile alternative to the slightly 
cheaper but riskier Discount Treasury Bills and the more expensive, but slightly less risky fixed interest bonds.

Figure 3: Optimal cost-risk frontier, composite risk based on 1/3 standard deviation - 1/3 ATR - 1/3 share of 1-year refinancing (corner compositions denote $100 \%$ financing compositions of a single instrument without liquidity costs or constraints)

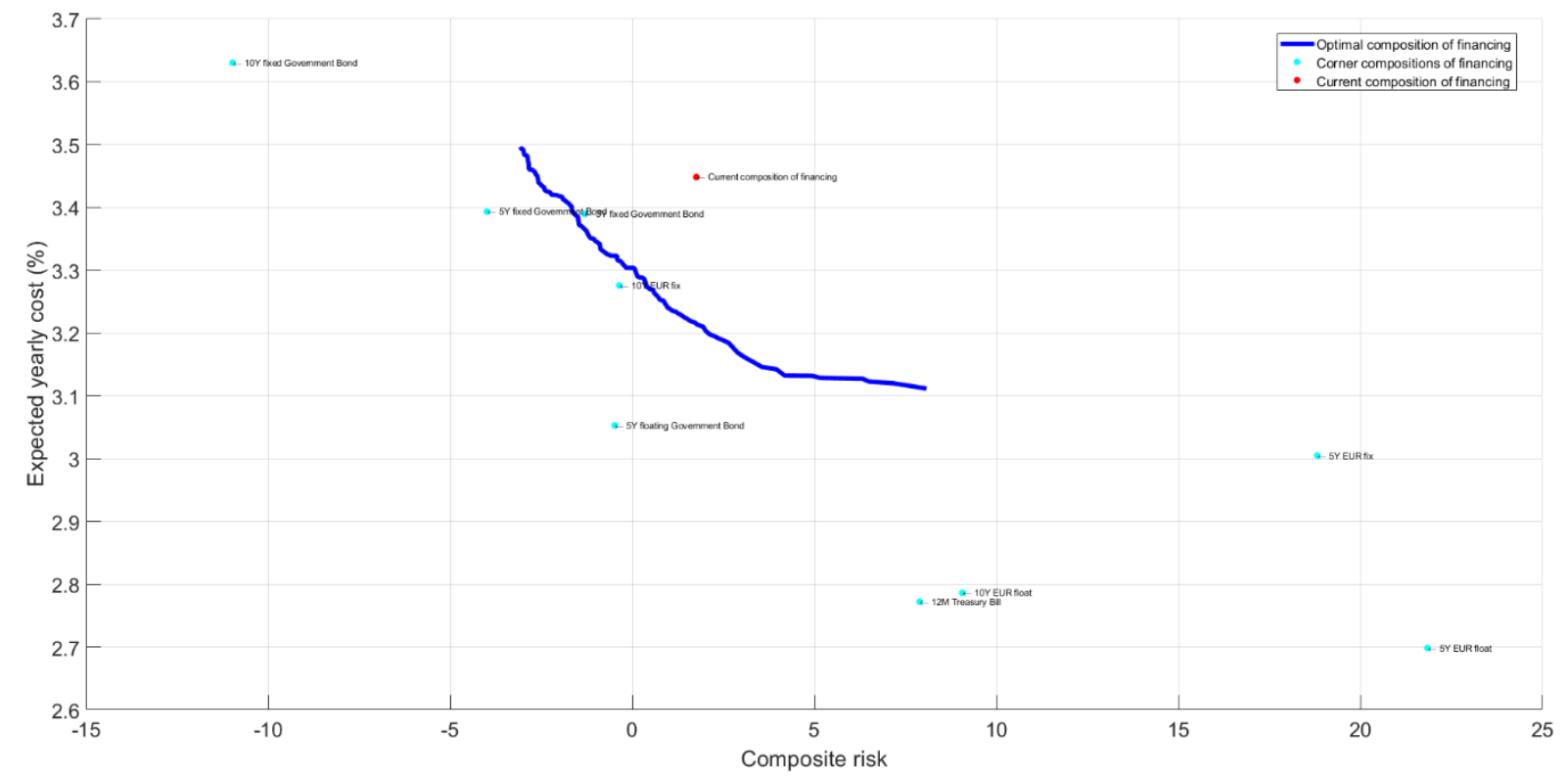

Source: Own calculations

Figure 3 shows the corresponding optimal cost-risk frontier. Current composition of financing means that during the simulation we intend to stick to the composition of the initial portfolio given at the start of the simulation as described in Section 2. The corner compositions of financing show the theoretical costs and risks associated with issuing a single debt instrument throughout the simulation. Similar to the optimal compositions of financing, corner compositions start from the initial portfolio. However, they take neither liquidity constraints nor liquidity costs into account.

We can deduce that the current financing composition is not optimal in this particular cost-risk framework of our stochastic model. The composition with the lowest risk would mean a reduction of 5 percentage points in the share of retail debt, substituting 3-year Hungarian Government Bonds with 5 and 10-year ones and issuing only 10-year fixed interest FX debt. The feasibility of such policy changes is of course a question, but the results can prompt the debt manager in a direction to achieve more optimal financing and debt portfolio structures.

\section{Conclusion}

In this paper we constructed a stochastic model to optimize the Hungarian government debt portfolio. The results of the multiobjective optimization can be used to help in constructing a medium term debt management strategy. According to the stochastic 
model the optimal cost-risk frontier shows that the current debt portfolio is not Paretooptimal and improvements can be considered.

We introduced several new concepts and techniques going beyond existing debt strategy models. Our model is the first to utilize a multiobjective approach for debt portfolio optimization. We also use historical data to model funding liquidity, including liquidity costs as well as constraints. Furthermore, our issuance algorithm uses a unique approach to move towards a desired debt portfolio as swiftly and efficiently as possible, while taking liquidity constraints into account.

The model has some limitations in order to reduce computing times to acceptable levels. One such limitation is that liquidity premia and yields are exogenous, and continued excess issuance has no long-term effect on the yield curve. The government deficit is also assumed to be exogenous and unaffected by debt expenditures.

The model is flexible enough to serve as a foundation for future analyses. For example, by removing or adding model instruments, we could measure the sensitivity and stability of the model and explore new possibilities such as financing the government using very long maturity or wholesale inflation-linked debt.Furthermore, the model can be used to carry out stress tests by examining the results of individual scenarios. Thus, the effects of a particularly severe shift in the yield curve, or the repercussions of a significantnegative demand shock could be analyzed.

\section{References}

Adamo, M., Amadori, A.L., Bernaschi, M., Chioma, C.L., Marigo, A., Piccoli, B., Sbaraglia, S., Uboldi, A., Vergni, D., Fabbri, P., lacovoni, D., Natale, F., Scalera, S., Spilotro, L., Valletta, A., 2004. Optimal Strategies for the Issuances of Public Debt Securities. International Journal of Theoretical and Applied Finance (IJTAF) 07, 805-822.

Balibek, E., Memis, H.A., 2012. Turkish treasury simulation model for debt strategy analysis (Policy Research Working Paper Series No. 6091). The World Bank.

Bebes A., Tran D., Bebesi L., 2018 (forthcoming). Yield Curve Modeling with Macro Factors: An Implementation of the Kim Filter in a Two-Economy Markov Regime Switching State-Space Model.

Bergström, P., Holmlund, A., Lindberg, S., 2002. The SNDO's Simulation Model for Government Debt Analysis.

Bolder, D.J., Deeley, S., 2011. The Canadian Debt-Strategy Model: An Overview of the Principal Elements (Discussion Papers No. 11-3). Bank of Canada.

Consiglio, A., Staino, A., 2012. A stochastic programming model for the optimal issuance of government bonds. Annals of Operations Research 193, 159-172.

Drehmann, M., Nikolaou, K., 2013. Funding liquidity risk: Definition and measurement. Journal of Banking \& Finance 37, 2173-2182. 
Fleiner T., Sziklai B., 2012. The nucleolus of the bankruptcy problem by hydraulic rationing. International Game Theory Review 14, 1-11.

Pick, A., Anthony, M., 2006. A simulation model for the analysis of the UK's sovereign debt strategy (UK Debt Management Office Research Paper).

Sun, H., Zhu, C., Cai, X., 2017. An Evolutionary Many-Objective Optimization Algorithm Based on Corner Solution Search. 